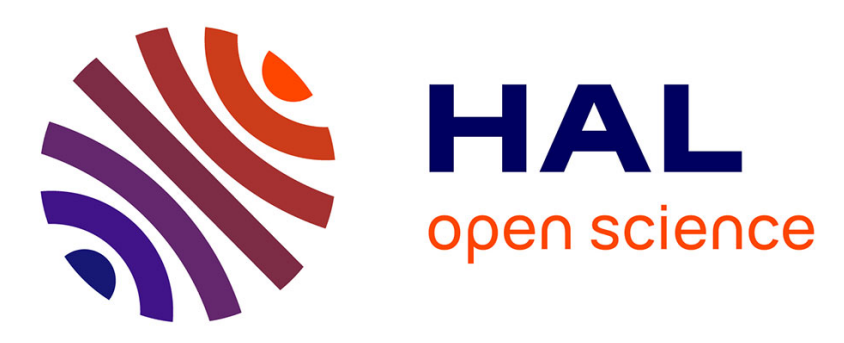

\title{
Les indices d'écologie du paysage à l'épreuve du marché foncier
}

Estelle Dumas, Ghislain Geniaux, Claude Napoleone

\section{To cite this version:}

Estelle Dumas, Ghislain Geniaux, Claude Napoleone. Les indices d'écologie du paysage à l'épreuve du marché foncier. 1. Rencontres du logement, Oct 2006, Marseille, France. 19 p. hal-02822986

\section{HAL Id: hal-02822986 \\ https://hal.inrae.fr/hal-02822986}

Submitted on 6 Jun 2020

HAL is a multi-disciplinary open access archive for the deposit and dissemination of scientific research documents, whether they are published or not. The documents may come from teaching and research institutions in France or abroad, or from public or private research centers.
L'archive ouverte pluridisciplinaire HAL, est destinée au dépôt et à la diffusion de documents scientifiques de niveau recherche, publiés ou non, émanant des établissements d'enseignement et de recherche français ou étrangers, des laboratoires publics ou privés. 


\title{
Les indices de l'écologie du paysage à l'épreuve du marché foncier
}

\author{
Estelle Dumas`, Ghislain Geniaux* et Claude Napoléone`
}

\section{Résumé :}

Les aménités paysagères jouant sur les choix individuels de localisation, sont généralement décrites dans la littérature économique en terme d'accessibilité aux aménagements paysagers urbains (un parc ou une forêt urbaine par exemple) ou aux espaces ouverts dans l'environnement immédiat des villes. Nous inscrivant dans la lignée des travaux de GEOGHEGAN et al. 1997, nous proposons une méthode couplant analyse hédonique et utilisation d'indices d'écologie du paysage, en nous appuyant sur une importante base de données des ventes des maisons individuelles spatialisées à l'échelle de la section cadastrale (département des Bouches du Rhône, 15000 ventes utilisés sur les 35000 ventes enregistrées entre 1990 et 2001). Cette démarche pluridisciplinaire nous permet de tester la gamme large de descripteurs des paysages périurbains et ruraux que fournissent les traitements géomatiques actuels des images satellitales de haute résolution, afin d'identifier ceux valorisés au sein du marché foncier.

\section{Summary:}

Landscape amenities acting upon location choice are generaly described in standard economic model, as urban green area or urban forest or suburb open space proximity. After GEOGHEGAN et al. 1997 works, we propose a method using both hedonic analysis and landscape ecology index. We use a large housing sale database, spatialised at cadastral level (Bouches du Rhône district, 15000 sales out of 35000 between 1990 and 2001). This pluridisciplinary method allows us to test some suburbs and rural landscape variables, given by current geomatic procedure, in order to identify these variables valued in residential market.

(integrating such knowledege may improve public policy about urbanisme or landscape)

Mots-clefs : Analyse hédonique spatialisée, indices d'écologie du paysage, marché foncier, préférences résidentielles.

JEL : R14, R21, Q240.

\footnotetext{
- IMEP-CNRS (Institut méditerranéen d'écologie et de paléoécologie), 6116 bâtiment Villemin. Europole de l'Arbois - BP 80 F. 13545 Aix en Provence cedex 04.

- INRA (Institut National de la Recherche Agronomique, Avignon FRANCE), GREQAM-IDEP (Groupement de Recherche en Economie Quantitative d'Aix-Marseille, Institut d'Economie Publique, Marseille).

- CEMAGREF (Centre d'Etude du Machinisme Agricole, du Génie Rural, des Eaux et des Forêts, Aix-en-Provence), IDEP (Institut d'Economie Publique, Marseille). Tel 0442669966 ; Fax : 0442669971. Adresse électronique : claude.napoleone@cemagref.fr
} 
De par ses choix d'occupation du sol, l'homme agit sur les fonctions écologiques des espaces naturels et influence directement les éléments qui déterminent l'appréciation qu'il peut porter par la suite sur leur «qualité paysagère». En retour, la qualité paysagère des milieux naturels ou partiellement artificialisés n'est pas sans conséquences sur les choix de localisation résidentielle. Pour rendre compte du dernier lien de cette boucle, le couplage entre modèles écologiques et économiques semble approprié. II est toutefois difficile d'identifier les indicateurs spatiaux qui soient pertinents d'un point de vue économique comme d'un point de vue écologique. D'autant plus que les modèles économiques ont du mal à rendre compte d'espaces aussi hétérogènes que peuvent l'être les milieux naturels d'une région ou d'un département. S'il est reconnu qu'il existe une capitalisation du «paysage» dans les prix immobiliers (SMITH et al. 2002, IRWIN et BELL 2001; LUTTIK 2000 ; GARROD et WILLIS 1992), la formalisation du lien entre le paysage et la valeur des biens immobiliers varie énormément dans les différents travaux sur ce domaine, notamment dans le choix des variables les mieux adaptées à «qualifier» le paysage. A la notable exception de GEOGHEGAN et al. 1997, on trouve essentiellement des études où le paysage se limite à un type particulier, comme la vue sur les forêts urbaines (TYRVAINEN 1997, MORANCHO 2003) ou la vue sur un parc public urbain (BOLITZER et NETUSIL 2000), conduisant à des évaluations à petite échelle où le rôle du paysage n'est pas abordé dans toute sa complexité. On retrouve également beaucoup de travaux abordant cette question, en référant uniquement la notion de paysage à la présence " d'espace ouvert ", c'està-dire non urbanisée (IRWIN et BELL 2001; GEOGHEGAN et al. 1997; BELL et BOCKSTAEL 1997). S'il est aisé de montrer quel est l'impact sur les prix des appartements ou des maisons lorsqu'on restreint la notion de paysage à un type de vue, il est beaucoup plus difficile d'identifier les «critères paysagers» valorisés au sein des marchés immobiliers et fonciers, pouvant s'appliquer à une large gamme de sites. Nous proposons dans cet article le couplage d'un modèle hédonique spatialisé et d'une batterie d'indicateurs de la qualité paysagère décrivant l'environnement des biens fonciers et immobiliers échangés, afin de mettre en évidence les critères paysagers agissant le plus significativement sur le marché immobilier d'un département français (les Bouches du Rhône).

Deux approches différentes de construction de ces indicateurs ont été évaluées. La première approche s'appuie sur la construction d'indices d'écologie du paysage. La seconde méthode s'appuie sur une meilleure appréhension de la dimension esthétique des paysages à travers des analyses à dires d'expert. Le couplage entre les indicateurs paysagers et les modèles hédoniques a été effectué en s'appuyant sur des données de ventes foncières et immobilières des Bouches-du-Rhône, spatialisées à la section cadastrale et d'indices paysagers agrégés à différentes échelles (de la section cadastrale à la commune). Nous avons ainsi pu apprécier l'ensemble des phénomènes majeurs concourant à la valorisation des biens fonciers et immobiliers et de replacer la contribution particulière de la qualité paysagère de l'environnement naturel dans le processus de valorisation des biens résidentiels. Un modèle box-cox a été utilisé pour l'estimation ainsi qu'une batterie de tests spatiaux permettant de vérifier les bonnes propriétés statistiques des estimateurs. Un travail particulier a été mis en œuvre pour sélectionner les formes fonctionnelles les mieux adaptées aux variables explicatives continues (nombre de pièces, surface habitable, surface terrain, distance-temps aux pôles d'emplois...) ; travail qui s'avère par ailleurs indispensable pour les indices paysagers du fait que la littérature ne fournit aucun point de référence.

\section{1) Préalables méthodologiques}

L'évaluation d'un bien non-marchand par des méthodes de révélations indirectes, comme la méthode hédonique, impose de vérifier deux préalables :

- disposer d'un descripteur correct du niveau de l'externalité à mesurer (le bien non-marchand),

- que le bien non-marchand mesuré ne fournisse ni services joints, ni ne puisse être "consommé » par d'autres voies que celle que considère l'observateur.

Sans ces pré-requis, les liens qui peuvent être mis à jour statistiquement entre les variations de prix et les variations du descripteur sont incorrects. Ces pré-requis sont particulièrement problématiques pour le paysage dont il n'existe pas a priori de descripteur unique, suffisant et spécifique. Dans pareil cas, l'approche hédonique fournit plus un outil d'investigation permettant de tester différentes caractérisations des biens non-marchands qu'un outil d'évaluation au sens strict. Le recours à la méthode hédonique pour identifier les variables permettant de «définir» un bien non-marchand n'est pas nouveau. Cette approche a été utilisée notamment dans le cadre de certaines nuisances ou 
externalités négatives comme le bruit ou la pollution. Dans le cas du bruit, on peut citer des études françaises récentes, menées au sein du programme PREDIT sur les nuisances sonores des aéroports (FABUREL et al. 2001) qui utilise la méthode hédonique pour tester différentes variables sensées caractériser les nuisances sonores. LESVEQUES 1994, dans une démarche comparable, a testé différents événements bruyants et différentes façons de les intégrer dans une fonction hédonique (moyenne ou écart-type des niveaux de bruits) : il montre que seule l'amplitude des niveaux de bruit mesurée par l'écart-type a une influence significative sur les prix des biens immobiliers, allant ainsi à l'encontre de l'idée que l'on s'habitue aux bruits des avions. On peut retenir de ce type de travaux sur l'identification des variables jouant dans la perception des externalités, des résultats de quatre ordres :

- Les formes fonctionnelles utilisées doivent être adaptées aux non-linéarités qui caractérisent généralement le fonctionnement des variables d'environnement, notamment sous la forme de seuils ou de paliers de perception.

- Les échelles spatiales et les niveaux de résolutions variant entre les données issues du marché immobilier et les mesures physiques des externalités, il importe de tester plusieurs modes d'agrégation des variables à l'échelle commune la plus fine possible et de resituer les différents modes d'agrégation par rapport à la littérature de la discipline particulièrement concernée par l'externalité observée (acoustique, épidémiologie, écologie, ....).

- Les externalités ne peuvent pas se limiter à une définition physique simple, mais intègrent différents phénomènes sociaux connexes : par exemple dans le cas du bruit, la visibilité des couloirs de vol ou des pistes participe à accroître la décote des biens immobiliers, soit parce qu'ils accentuent la perception, soit parce qu'il ajoute à la gêne des perceptions de risques supplémentaires (accidents, pollution atmosphérique,...) (FABUREL et MALEYRE 2002). Par exemple, dans le cas du paysage, une vue dominante renvoie à deux notions : la vue et la dominance et il reste difficile de séparer ce qui revient au paysage de ce qui revient à des phénomènes de distanciation sociale.

- Une estimation valide des effets associés à différentes variables de paysage demande que la méthode hédonique soit appliquée avec rigueur pour l'ensemble des phénomènes pouvant avoir de l'influence sur les prix, ou du moins s'attache à tenir compte des phénomènes les plus significatifs sur le marché immobilier. Autrement dit, les investigations doivent porter sur le fonctionnement du marché immobilier dans son ensemble, et les estimations économétriques doivent répondre à un certain nombre d'exigences méthodologiques. Ainsi, les estimations doivent s'appuyer sur une base de données importante en termes quantitatif et qualitatif, c'est-à-dire le nombre d'observations de ventes, mais également en termes de diversité et de qualité des variables renseignant les transactions et leur environnement.

Au-delà de ces préalables méthodologiques, nous nous sommes situés dans un cadre exploratoire où la confrontation de différentes définitions objectives du paysage au sein d'un modèle hédonique de formation des prix immobiliers, nous permet d'évaluer leur capacité à rendre compte de la capitalisation foncière. Ainsi, plus le lot de variables décrivant le paysage est statistiquement significatif au sein du modèle, plus la définition associée à ce lot de variables se rapproche de la notion de paysage que les individus considèrent dans leur décision d'achat résidentiel. Insistons ici sur le fait que la procédure ne valide pas une notion de paysage au sens large mais une notion de paysage contextuelle et valable principalement dans le contexte de la localisation résidentielle ${ }^{1}$.

II est à noter que la démarche pluridisciplinaire que nous présentons, nécessite également une adaptation des pratiques et des modèles des écologues associés. En premier lieu, ces derniers ont consenti à adopter un terrain d'étude marqué par la présence de bâti, alors que généralement leurs travaux s'en éloignent pour éviter au maximum les perturbations anthropiques. En second lieu, ils ont intégré la structure spatiale du bâti (dense vs diffus) dans leurs modèles, afin d'estimer les influences des formes d'urbanisation sur les formes paysagères.

\footnotetext{
${ }^{1}$ Cela mériterait de ce point de vue, une discussion spécifique sur la façon dont les individus peuvent concevoir, faire usage ou s'approprier un paysage dans le cas de la propriété privée d'une maison d'habitation, discussion qui va au-delà du cadre de ce présent article.
} 


\section{2) Mode de construction des données "paysagères » 2.1) Indices d'écologie du paysage.}

Les développements récents de l'écologie du paysage permettent de caractériser les paysages à partir d'une batterie d'indices quantifiés reposant sur l'analyse de la structure spatiale d'une zone quelconque. Les mesures de l'hétérogénéité, de la fragmentation et/ou de la connectivité des espaces sont généralement utilisées et ont toutes des incidences établies sur le fonctionnement des écosystèmes (BAUDRY et BUREL 2000). Ces indices fournissent un moyen d'agréger à différentes échelles l'information sur la structure de l'occupation du sol et renseignent à la fois sur les dynamiques naturelles et sur les modes d'occupation de l'espace à des niveaux très fins (WICKHAM, O'Neill et Jones, 2000, CHOPPING et HAINES 1997). On peut ainsi envisager des fenêtres de l'ordre de 300 mètres sur 300 mètres, qui constituent dans le périurbain une échelle beaucoup plus fine que la section cadastrale (Les données de ventes sont spatialisées à l'échelle de la section cadastrale).

Pour chacune des classes d'indices (fragmentation, connectivité et hétérogénéité), la littérature fournie une multitude d'indices spécifiques. La question de la formulation la plus pertinente des indices utilisés est donc largement contingente au type de fonctionnement écologique étudié et/ou au type d'informations spatiales recueillies. Seul l'indice de diversité de Shannon fait relativement consensus dans la mesure où ses propriétés répondent formellement à la liste d'axiomes souhaités pour rendre compte de la notion de diversité.

A partir d'une catégorisation en 12 classes de couverts et d'occupation du sol', nous avons estimé une gamme de 6 types d'indices : la proportion de la surface occupée par chacune des 12 classes d'occupations du sol $\left(P L A N D_{i}\right)$ l'indice de Shannon $(S H D I)$, , l'indice d'Hétérogénéité interclasses $\left(H_{i j}\right)$, l'indice d'agrégation (AI), l'indice d'interspection et de juxtaposition (IJI) et l'indice de richesse de taches $(R P R)$.

Formellement, on a :

- $\quad P L A N D_{i}=p(i)=\sum_{j} a_{j} / A * 100$, avec $a_{i}$ la surface en $m^{2}$ de la tache d'occupation du sol $i$ et $A$ la surface de la section. $P L A N D_{i}$ correspond au pourcentage de classe $i$ dans la section cadastrale.

- $S H D I_{f}=-\sum_{i} p(i) \ln (p(i))$ qui représente l'indice global de diversité de Shannon, calculé à partir des proportions $p$ (i) de chaque classe d'occupation du sol, pour des fenêtres $f$ de 300 mètres sur 300 mètres soit 9 ha. On utilise alors des statistiques estimées à l'échelle de la section cadastrale (l'indice moyen, l'indice moyen pondéré par la surface, l'indice médian, le coefficient de variation, le rang et l'écart type de l'indice).

- $\quad H_{i j}=-\sum p(i, j) \ln (p(i, j))$ à l'échelle de la section avec $p(i)=$ proportion de classe (i) en considérant les classes d'occupation du sol deux à deux, $i$ et $j$ dans notre formulation (on utilise alors les catégories regroupées en grande classe d'occupation du sol: urbain, agricole et forêt).

- $\quad A I=\left[\sum_{i=1}^{m}\left(\frac{g_{i i}}{\max \rightarrow g_{i i}}\right) \operatorname{PLAND}_{i}\right](100)$ avec $g_{i i}=$ nombre de contacts entre les différentes tâches d'une même classe d'occupation du sol $i$ au sein d'une fenêtre, rapporté au nombre maximal de contacts intra-classes observé sur l'ensemble du paysage.

- $\quad I J I=\frac{-\sum_{i=1}^{m} \sum_{k=i+1}^{m}\left[\left(\frac{e_{i k}}{E}\right) \bullet \ln \left(\frac{e_{i k}}{E}\right)\right]}{\ln (0.5[m(m-1)])}(100)$ avec $e_{i k}=$ longueur totale des contacts entre deux classes d'occupation du sol $i$ et $k\left(e_{i k}\right)$, rapporté à la longueur totale des contacts entre classes différentes sur un paysage $(E)$ et pondéré par le nombre de contacts observés $(m)$.

- $\quad R P R=m / m_{\max }{ }^{*} 100$ avec $\mathrm{m}$ le nombre de classes de la section cadastrale et $m_{\max }$ le nombre de classe maximum ( 12 dans notre cas).

\footnotetext{
${ }^{2}$ Territoires agricoles, vignoble, territoires a dominante agricole avec présence d'habitations résidentielles, milieux à végétation arbustive et/ou herbacée, forêt, plan d'eau, tissu urbain continu, tissu urbain discontinu, bâti isolé, zones industrielles ou commerciales et réseaux de communication, mines, décharges et chantiers et autres territoires
} 
Lors de l'intégration de ces indices d'écologie du paysage à un modèle hédonique, certaines questions méthodologiques s'imposent sur les niveaux d'agrégation. II existe, pour une fenêtre donnée, des indices concernant les taches (zones non-disjointes de même classe), des indices concernant les classes (une classe regroupe l'ensemble des tâches de même classe au sein d'une fenêtre) et enfin des indices concernant le paysage global (les indices de paysage sont calculés pour l'ensemble de la fenêtre et pour toutes les classes). Le couplage entre modèles économiques et indices d'écologie du paysage, révèlent trois difficultés :

- L'agrégation entre classes constitue une perte d'information si elle se fait à une échelle équivalente à celle de la variable endogène.

- Calculer des indices de diversité s'appuyant sur les superficies des taches d'occupation du sol, à partir d'un canevas spatial hétérogène ne permet pas statuer sur la variance observée, entre l'effet "indice" et l'effet "taille". Or, les découpages socio-économiques sont souvent hétérogènes en taille.

- Les indices de paysage tel qu'on les retrouve généralement dans la littérature en écologie ne laissent pas l'opportunité de considérer différemment certains types de couverts, si ce n'est en modifiant les catégories elles-mêmes, autrement dit en rejetant certaines catégories de l'analyse lorsqu'on agrège entre classes. Alors que l'analyse économique peut nécessiter de comparer les rôles particuliers de certaines classes d'occupation de l'espace et de couvert dans leur participation à la structuration du paysage.

Pour tenir compte de l'ensemble de ces contraintes, nous avons adopté une démarche intégrant les indices paysagers sous différentes formes:

- Sous forme d'indices estimés à l'échelle de la section cadastrale : c'est le cas pour les indices $S D H I, R P R, A l, I J I$, et $P L A N D_{i}$ pour $i=1$ à 12 . Ces indices sont notés avec un préfixe $G$ : GSHDI, GRPR, GAI, GIJI et GPLAND,

- Sous forme agrégée entre catégories à partir de fenêtres de taille inférieure à celle de l'échelle de spatialisation des données économiques, c'est-à-dire inférieure à la section cadastrale. La fenêtre qui s'est avérée la mieux adaptée est une fenêtre de 300 par 300 mètres. On estime ensuite, différentes statistiques représentant la distribution des indices du paysage sur chaque section cadastrale : l'indice moyen (préfixe $m$ ), l'indice moyen pondéré par la surface (préfixe $m p$ ), l'indice médian (préfixe $m d$ ), le coefficient de variation (préfixe $c v$ ), le rang (préfixe $r g$ ) et l'écart type de l'indice (préfixe $s d$ ) : c'est le cas pour les indices $S H D I, H_{i j}{ }^{3}$. Ces indices sont notés : $m S H D I, m p S H D I, m d S H D I, c v S H D I, r g S H D I$, sdSHDI, $m H_{12}, \mathrm{mpH}_{12}, \mathrm{mdH}_{12}, \mathrm{cvH}_{12}, \mathrm{rgH}_{12}, \mathrm{sdH}_{12}, \mathrm{mH}_{13}, \mathrm{mpH} \mathrm{H}_{13}, \mathrm{mdH}_{13}, \mathrm{cvH}_{13}, \mathrm{rgH}_{13}$, $s d H_{13}, \mathrm{mH}_{23}, \mathrm{mpH}_{23}, \mathrm{mdH}_{23}, \mathrm{cvH}_{23}, \mathrm{rgH}_{23}, \mathrm{sdH}_{23}$.

Deux types d'images ont été testées pour estimer ces indices :

- Les images aériennes numérisées issues de vol photogramétrique (BD Ortho de l'IGN). Ces prises de vues verticales en vraies couleurs (IGN 1/25 000), proche infrarouge (IFN 1/17 000) ou en niveaux de gris sont effectuées à une altitude constante. Chaque photo recouvre la précédente à $80 \%$. Ces données posent des problèmes d'analyse automatisée :

- Des phénomènes de sur- et sous- exposition modifient localement la luminosité et perturbent la radiométrie des objets.

- Les prises de vues aériennes ne pouvant être effectuées en simultanée sur la totalité d'un département, direction et taille des ombres projetées varient considérablement selon l'heure d'acquisition de la photographie. Les textures de l'image s'en trouvent profondément modifiées, et il devient difficile de traiter de grandes surfaces.

- Les images satellitales (Spot, Landsat, Ikonos, Quickbird). Un satellite dit à Très Haute Résolution Spatiale comme Quickbird fournit plusieurs images, chaque image ou scène couvrant une surface de $16,5 \mathrm{~km}$ sur $16,5 \mathrm{~km}$ :

\footnotetext{
${ }^{3}$ Pour $H_{i j}$, nous nous sommes appuyés sur une classification agrégée à 5 catégories et n'avons construit ces indices d'hétérogénéité interclasse que pour les croisements entre 3 des 5 catégories (Territoires artificialisés $i, j=1$, Territoires agricoles $i, j=2$, Forêts et milieux semi-naturels $i, j=3$ ).
} 
- Une image représentée en 256 niveaux de gris captée par le canal panchromatiques qui enregistre la bande spectrale de 0,45 à $0,90 \mu \mathrm{m}$, et ayant une résolution de $0,7 \mathrm{~m}$ (le pixel ou plus petite cellule de l'image est un carré de $0.7 \mathrm{~m}$ de côté)

- Une image multispectrale avec l'enregistrement de 4 bandes radiométriques B1 (Bande Bleue) de 0,45 à 0,52 $\mu \mathrm{m}, \mathrm{B} 2$ (Bande Vert) de 0,52 à 0,60 $\mu \mathrm{m}, \mathrm{B} 3$ (Bande Rouge) de 0,63 à $0,69 \mu \mathrm{m}$ et B4 (Bande Proche Infra Rouge) de 0,76 à $0,90 \mu \mathrm{m}$ ) ayant une résolution de $2,8 \mathrm{~m}$.

Au-delà de la simple limite due à la précision, de multiples effets viennent bruiter le signal lumineux reçu par les capteurs du satellite (les effets atmosphériques, d'angle de prises de vue, d'angle d'élévation solaire, du relief et de masque). Si l'on veut « donner du sens » à l'image brute et détecter des objets, il faut donc effectuer une classification. Le but est de regrouper des ensembles de pixels similaires en classes ; il existe deux méthodes générales : la classification non supervisée et la classification supervisée ou classification par maximum de vraisemblance. Cette dernière est majoritairement utilisée et se déroule généralement en quatre phases :

1- sélection d'échantillons (ou parcelles d'entraînement) sur l'image;

2- évaluation de la qualité des échantillons ;

3- classification par maximum de vraisemblance : affectation des pixels aux échantillons les plus proches selon une distance basée sur la probabilité d'un pixel d'appartenir à une classe donnée calculée entre le pixel à classer et chaque parcelle d'entraînement ;

4- évaluation de la classification: cette évaluation se fait visuellement en comparant la classification à d'autres données externes (carte, photographie) ou statistiquement en regardant comment tel ou tel échantillon non pris en entrée est classé en sortie par la classification (construction des matrices de confusions).

Nous présentons ici les résultats s'appuyant sur les indices calculés à partir des proportions de 12 types de couverts ${ }^{4}$ reconstitués à partir d'analyses spectrales de photos satellitales sur l'ensemble des Bouches-du-Rhône, réalisés à partir de photos satellitales LANDSAT précises à $30 \mathrm{~m}$ (voir exemple figure 1).

\section{[FIGURE 1]}

\section{2) L'ambiance paysagère}

L'ambiance paysagère correspond à l'objectivation de la nature et de l'agencement des éléments paysagers, réalisée à dire d'experts (FISCHESSER et DUPUIS-TATE 2001). En se référant à la neurophysiologie, on estime qu'un paysage est perçu d'emblée dans son ensemble (notion d'organisation spatiale et d'échelles), puis que l'observateur passe ensuite à l'analyse de détails et y distingue des zones homogènes différenciées par leurs composantes visuelles (lignes, formes, couleurs, textures...). Une appréciation inconsciente des relations entre éléments visuels et leurs effets de valorisation ou de dévalorisation réciproque entre eux et par rapport à l'ensemble du paysage, permet à l'observateur de ressentir l'ambiance dégagée par une unité paysagère (KIEMSTEDT 1967; DE COULON 1988).

L'évaluation à dire d'experts présente l'avantage de correspondre aux méthodologies les plus fréquemment utilisées dans la mise en œuvre de procédures publiques (inventaires paysagers, plan d'occupation des sols, etc.). De plus, elle est susceptible de rendre compte assez finement d'éléments subjectifs (esthétiques par exemple) qui entrent dans le choix des individus. Cependant, elle présente l'inconvénient d'être difficilement intégrable à une analyse économique quantifiée. Afin de disposer d'informations compatibles entre l'expression d'une subjectivité et notre visée généralisante, nous avons retenu une démarche empirique, avec des hypothèses de départ relativement fortes, mais dont les résultats peuvent être testés dans les modèles économétriques. Concrètement, nous avons sélectionné des lots de sections cadastrales sur quatre sites d'études en utilisant d'une part l'approche fonctionnaliste et d'autre part en faisant l'hypothèse que le paysage puisse être un bien supérieur :

\footnotetext{
${ }^{4}$ Territoires agricoles, vignoble, territoires a dominante agricole avec présence d'habitations résidentielles, milieux à végétation arbustive et/ou herbacée, forêt, plan d'eau, tissu urbain continu, tissu urbain discontinu, bâti isolé, zones industrielles ou commerciales et réseaux de communication, mines, décharges et chantiers et autres territoires
} 
- La démarche fonctionnaliste considère qu'un territoire (généralement une agglomération urbaine) est un ensemble d'espaces ayant chacun des fonctions propres. Trois principaux types de fonctions sont ainsi généralement attribués aux espaces d'une agglomération: résidentielle, productive ou ludique (LE CORBUSIER 1971 ; RONCAYOLO 1999; GÉRARD-VARET 2000). Pour simplifier, des éléments de base comme les densités résidentielles ou le degré d'artificialisation des sols sont à même d'établir une différence entre les grandes vocations spatiales observables (du quartier urbain à la commune "rurale") et sont utilisables dans une analyse économique développée sur un département ou une agglomération urbaine ${ }^{5}$.

- L'hypothèse que le paysage puisse être un bien supérieur peut être posée lorsqu'il s'agit d'analyser les comportements résidentiels. Nous faisons simplement l'hypothèse que les ménages les moins riches privilégient des éléments comme la superficie habitable ou l'éloignement au centre, par rapport à la jouissance d'aménités paysagères. Dans cette perspective, nous avons choisi de retenir les sections cadastrales où s'étaient effectuées les ventes de maisons les plus onéreuses, en postulant que ces sections cadastrales ont une probabilité plus grande d'être caractérisées par une qualité paysagère supérieure.

Chaque site d'étude sélectionné a ensuite été présenté aux experts, en leur proposant d'objectiver un nombre réduit de caractéristiques qui leur seraient communes. La seule contrainte imposée fut que les caractèristiques puissent être estimés quantitativement et automatiquement (par SIG) sur l'ensemble des sections cadastrales du département. Quatre critères paysagers étaient présents dans chacune des situations (FISCHESSER et DUPUIS-TATE 2001) :

- La profondeur de champ mesure les superficies visibles à partir de chaque section cadastrale et en prenant en compte le relief. II est à noter que la littérature de référence en analyse paysagère, spécifie un seuil de $5 \mathrm{~km}$ comme une limite entre vision de proximité et "échappée visuelle".

- La dominance. Elle rend compte à la fois d'un critère paysager de par les vues longues qu'elle permet, mais également d'un comportement social de distanciation qui rend préférable les situations dominant le voisinage par rapport aux autres possibilités de localisation.

- La texture rend compte de "l'inconfort visuel" inhérent à la juxtaposition sans ordre d'occupations différentes du sol. La texture peut être caractérisée par exemple par les différents types d'occupation du sol (une forêt de haute futée, une agriculture de grande culture...) et leur structure dans l'espace.

- L'exposition enfin, puisque dans la zone d'étude choisie l'orientation au sud protège des vents dominants, maximise l'ensoleillement.

Nous avons alors estimé différents indices permettant de rendre compte de ces 4 critères paysagers sur l'ensemble du département. Nous présentons ici les résultats obtenus à partir de l'intégration au sein des modèles économétriques, de six variables calculées à l'échelle de la section cadastrale :

- PAlti. L'altitude du barycentre de la section cadastrale.

- PDomin. La dominance de la section cadastrale, calculée par différence entre l'altitude du barycentre de la section et l'altitude du centre villageois.

- $\quad$ PVisiTot. La visibilité totale à partir du barycentre de la section cadastrale, soit le nombre de pixels visibles multiplié par leur taille.

- PVisi5000. La visibilité dans un rayon de moins de $5 \mathrm{~km}$, à partir du barycentre de la section cadastrale.

- PVisi5000sup. La visibilité au-delà d'un rayon de $5 \mathrm{~km}$, à partir du barycentre de la section cadastrale.

- Porient. L'orientation de la section par rapport au sud (exprimée en degrés).

- Tissu urbain discontinu. Le pourcentage sectionnal de la catégorie d'occupation du sol " tissu urbain discontinu » qui représente un indicateur (partiel) du phénomène de mitage et de la texture du paysage.

\footnotetext{
${ }^{5}$ En l'occurrence, nous avons sélectionné une commune urbaine, une commune rurale, une commune résidentielle et une commune industrielle.
} 
L'organisation des paysages au sein du département que permet d'identifier la répartition géographique de ces indices, qui sort du cadre de cet article, est plus longuement développée dans GENIAUX et al. 2003.

\section{3) Les données disponibles}

Sur le périmètre de l'agglomération marseillaise, et par extension sur le département des Bouches-du-Rhône, nous avons constitué une base de données à partir de l'ensemble des informations disponibles sur la question foncière (tableau 1).

\section{[TABLEAU 1]}

Nous disposons pour mettre en œuvre des modèles hédoniques des marchés fonciers et immobiliers :

- De données sur le marché foncier et immobilier sur une période de 10 ans : une centaine de milliers de ventes de terres agricoles (33 074 obs.), ventes immobilières (83026 obs.) et permis de construire, renseignés sur 24 à 60 caractéristiques. Les données regroupées représentent la quasi-exhaustivité des données disponibles sur le marché foncier dans les Bouches-du-Rhône ${ }^{6}$.

- De données sur l'environnement de la vente à l'échelle de la section ou de la commune : ces données regroupent des informations à la fois sur les biens publics locaux, sur l'environnement social, sur l'usage du sol (stock de logements ou mises en constructions nouvelles par exemple), sur la fiscalité et les règles d'urbanisme, sur les espaces naturels.

- D'informations géographiques : un ensemble de cartes numérisées qui permettent de situer chaque vente dans son environnement physique (les réseaux d'échanges, l'occupation du territoire, la composition sociale des quartiers, etc.).

- Enfin, pour lier les bases de données quantifiées aux différentes cartes à notre disposition, nous avons numérisé les 4929 sections cadastrales du département (à chaque vente correspond une adresse composée du nom de la commune et d'un code alphanumérique spécifique à chaque section). Beaucoup plus précises qu'une commune, la taille des sections cadastrales est fonction du nombre d'individus y résidant : une section urbaine sera très petite, une section rurale sera plus grande.

La liaison entre ces différents types de données est assurée par $\mathrm{SIG}^{7}$, ce qui nous donne la possibilité de calculer des données spatialisées quantifiées rentrant dans la définition de la fonction de prix hédonique. Par exemple, estimer le temps de transport nécessaire pour accéder à divers pôles d'emploi à partir du barycentre de la section cadastrale où s'est réalisée la vente. Souhaitant toutefois nous focaliser sur les critères paysagers non urbains, nous avons restreint notre analyse au contexte périurbain et rural en centrant notre travail sur le marché des maisons individuelles (25000 ventes). Nous avons également exclu les types de maisons très spécifiques comme les fermes, les chalets ou encore les hôtels particuliers, mais également les observations qui n'étaient pas correctement renseignées sur l'ensemble des variables explicatives utilisées dans les modèles et correctement spatialisées (absence ou problème de codage de la section cadastrale): les modèles sont ainsi estimés sur environ 15000 observations de vente entre 1991 et 2001.

\section{4) Le modèle}

L'analyse économétrique des observations de vente a été effectuée sur les logiciels SAS et $R$ en utilisant une modélisation hédonique s'appuyant sur un modèle Box-Cox du type suivant :

$$
P(\lambda)=\alpha+\beta X_{C}+\theta X_{V E}+\delta X_{V P}+\varepsilon
$$

\footnotetext{
${ }^{6}$ La chambre départementale des notaires, génératrice des données que nous avons utilisées, estime qu'elle collecte approximativement $90 \%$ des ventes réalisées ; cette collecte reposant sur le volontariat des différents notaires.

${ }^{7}$ Utilisant le logiciel Arc Info.
} 
Où $P(\lambda)=\frac{\frac{P^{\lambda}-1}{\lambda}}{\dot{P}^{\lambda-1}}$ pour $\lambda \neq 0$ et $\dot{P}$ représente la moyenne géométrique de $P$ et $X_{c}$ regroupe l'ensemble des variables explicatives décrivant les caractéristiques du bien immobilier et de la transaction, et $X_{V E}$ regroupe les variables renseignant l'environnement économique et social du bien et $X_{V P}$ regroupe les variables et indices paysagers.

Le processus de spécification mis en œuvre s'appuie sur une procédure OLS robuste à l'hétéroscédasticité et, outre les tests classiques, a mobilisé des tests d'hétéroscédasticité (voir DAVIDSON et MACKINNON 2004) et des tests spatiaux : Moran I, $\mathrm{LM}_{\mathrm{lag}}$, $\mathrm{LM}_{\mathrm{lag}}{ }^{*}, \mathrm{LM}_{\mathrm{err}}$, et $\mathrm{LM}_{\text {err }}{ }^{*}$ (voir ANSELIN et al. 1996). En effet, la nature spatialisée des biens fonciers est à l'origine de problèmes d'indépendance des résidus au niveau spatial dans les régressions hédoniques et nécessite ce type de tests. Ces problèmes sont dus au fait que dans un voisinage, les biens fonciers sont soumis aux mêmes facteurs environnementaux et affichent souvent des caractéristiques structurelles identiques. Si ces facteurs influent sur les prix de vente alors toute erreur dans leur mesure est susceptible d'entraîner de l'autocorrélation spatiale et/ou de l'hétérogénéité spatiale (ANSELIN 1988, CAN 1990, DUBIN 1998). On peut également faire face à des régimes spatiaux différents qui se caractérisent par des modes de comportement résidentiel structurellement différents dans différentes zones du département. Les tests spatiaux ont été effectués avec différentes matrices de poids spatiaux (voisinage à 5,10 et $20 \mathrm{~km}$, contiguïté des communes ou sections à l'ordre 1,2 ou 3, inverse de la distance) sur des échantillons d'observations uniquement ${ }^{8}$. Nous avons également testé la présence de régimes spatiaux différents entre Est et Ouest du département et entre les différentes zones ${ }^{9}$ de marché identifiées par la chambre départementale des notaires.

La démarche générale retenue a été de partir d'un modèle intégrant l'ensemble des variables $X_{c}$ correctement renseignées sur les marchés analysés n'incluant aucune variable paysagère. Nous avons ensuite spécifié un modèle Box-cox restreint $\left(X^{*} \subset X\right)$ et particulièrement travaillé sur les formes fonctionnelles adaptées aux variables de surface. La présence d'autocorrélation spatiale, de normalité des résidus et d'hétéroscédasticité a été évaluée à ce stade. Nous avons ensuite intégré les variables $\mathrm{X}_{\mathrm{VE}}$ et $\mathrm{X}_{\mathrm{VP}}$ au regard de leur significativité et en contrôlant leur contribution aussi bien à réduire les problèmes d'autocorrélation spatiale qu'à créer des problèmes de multicollinéaritée ${ }^{10}$. Les tests spatiaux nous ont conduit à écarter de nos données les maisons individuelles vendues dans les villes de Marseille et de Aix en Provence qui ont des comportements très spécifiques et introduisaient des problèmes importants d'hétérogénéité spatiale et de non-normalité des résidus (figure 2). On notera qu'écarter les ventes sur ces deux villes n'élimine naturellement pas leurs effets d'attractivité sur les ventes dans les communes alentours.

\section{5) Résultats et commentaires}

Deux types de modèles sont ici proposés : un modèle spécifié à partir de l'ensemble des informations disponibles (M1) et un modèle où seules les variables paysagères, en plus de caractéristiques de la maison, sont candidates dans le processus de spécification du modèle (M2). Les variables paysagères sont en effet corrélées avec toute une gamme de variables d'environnement économique et social. Comme il n'est pas possible d'identifier l'ensemble des processus de cause à effet dans le processus de localisation résidentielle, il peut être éclairant de disposer de ces deux informations (M1 et M2), afin de mettre en évidence l'effet marginal des différentes variables paysagères selon que l'on tienne compte ou pas des effets des autres facteurs de différentiation de l'espace. Pour les deux modèles, les transformations de Box-Cox donnent des paramètres autours de 0.3 pour la dépendante et de 0 (transformation log) pour les variables explicatives surface de l'habitation et surface du terrain.

\section{[TABLEAU 2]}

Concernant le modèle M1 (voir tableau 2), malgré un nombre limité de variables explicatives ainsi qu'une une large gamme de biens immobiliers considérés que ce soit en termes de caractéristiques

\footnotetext{
${ }^{8}$ Nous avons effectué les tests spatiaux en sélectionnant une série d'échantillons d'environ 1000 observations sur lesquelles les tests spatiaux sont mis en œuvre. Les résultats proposés reprennent, pour chaque matrice de poids et pour chaque statistique de test, les résultats de l'échantillon le plus propice au rejet de l'hypothèse nulle.

${ }_{9}^{9}$ On retrouve les 7 zones en question dans les journaux d'annonces immobilières qui segmentent ainsi souvent les offres.

${ }^{10}$ Utilisation de l'indicateur VIF (Variance Inflation Factor).
} 
propres que de l'échelle spatiale, on obtient un très bon ajustement du modèle avec un R2 ajusté de 0,73 . Par rapport à l'ensemble des variables disponibles dans notre SIG et candidates dans le processus de spécification du modèle (M1), on notera que de nombreuses variables significatives par OLS sont rejetées en utilisant une OLS robuste à l'hétéroscédasticité, aussi bien pour ce qui concerne les variables d'environnement du bien que pour les caractéristiques propres aux maisons. Par ailleurs, le contrôle à travers le critère VIF des problèmes de colinéarité conduit à une sélection drastique dans les variables d'environnement renseignant les proximités à des pôles ou des services publics ou le contexte socio-économique au niveau communal ou intercommunal (fiscalité, biens publics locaux, richesse et composition sociale, ...). Ainsi, on remarque dans le tableau 2 une très forte dominance des caractéristiques de surface, du type de maison, et de l'année de vente. Cela témoigne assez clairement d'une plus forte importance des caractéristiques internes du bien que des variables d'environnement. Pour valider cette hiérarchie, nous avons procédé à des tests de significativité par groupe de variables afin de hiérarchiser les différents critères de valorisation des maisons individuelles. Les statistiques présentées dans le tableau 3 correspondent à un test de nullité conjointe de l'ensemble des variables explicatives du groupe de variables considéré en gardant l'ensemble des autres groupes au sein du modèle (M1).

\section{[TABLEAU 3]}

On remarque également dans le tableau 3 une importance majeure de la richesse globale des ménages de la commune et des effets de certaines communes ou de zones de marché. La richesse communale peut être à l'origine de problème d'endogénéité et demanderait une estimation du modèle par variables instrumentales. Les effets de certaines communes ou de zones de marché ne peuvent être capturés, à ce stade de nos travaux, ni par les variables de richesse et de fiscalité ou de composition sociale de la commune, ni par leur situation en termes de distance aux pôles, ni par des variables paysagères. Les paramètres estimés associés à ces variables de localisation spatiale peuvent être interprétés comme des effets de notabilité trouvant leur origine dans l'absence de certains descripteurs (inexistants ou inobservables) et visent à capturer les effets spatiaux nuisibles dans le processus de spécification du modèle (M1). Ainsi, l'intégration des variables spatiales muettes permet, en capturant des valeurs de zone (communale ou par groupe de communes), de réduire une autocorrélation spatiale des résidus opérant à l'ordre de la commune et des communes contiguës comme l'atteste, en annexe, la comparaison des cartes proposées (figure 2) et des tests spatiaux (tableau 5 et 6). On constate ainsi l'importance que revêt l'identification de descripteurs spatiaux permettant de mieux appréhender la répartition spatiale de la valeur immobilière des maisons.

Les variables paysagères sont significatives et affichent, dans le tableau 3 , un effet global dominant celui des variables de distance. Les variables de distance/temps jouent de façon linéaire sur les prix avec un effet positif de la proximité au plus gros pôle d'emploi et de services qu'est Marseille, et un effet négatif de la proximité à une autoroute (distance euclidienne) qui traduit ainsi un effet dominant des nuisances de ce type d'infrastructure routière pour les sections très proches. Au sein des variables paysagères, peu de variables à dire d'expert s'avèrent significatives, et seule l'orientation de la section par rapport au sud de la section fournit des résultats aisément interprétables. Pour les variables d'écologie du paysage, on constate en premier lieu que les variables décrivant la proportion des différentes classes d'occupation du sol jouent très fortement, notamment celles correspondant aux zones de mitage (bâti isolé, territoire à dominante agricole avec présence d'habitat résidentiel) et aux espaces urbains denses. L'effet significatif de la classe Tissu Urbain Continu illustre bien la pauvreté du critère distance au centre pour rendre compte de la proximité à de l'urbain. L'intégration dans les modèles hédoniques des pourcentages de Tissu Urbain Continu par section cadastrale permet précisément une meilleure appréciation de l'effet de la distance aux différents pôles d'emploi et de services. Contrairement à une idée reçue, la forêt immédiate n'est pas un critère valorisé de façon univoque dans la localisation résidentielle, puisque le taux de couvert forestier joue négativement sur les prix. On a un effet négatif des zones à très forte proportion de forêt qui domine largement les effets positifs des zones où il y a des répartitions plus homogènes entre forêt et autres types de couvert. L'effet positif de la forêt est essentiellement dû aux interfaces forêt/urbain et peut se lire dans l'indice d'hétérogénéité interclasse entre zone artificialisées et forêt (Moyenne sectionnale de $\left.\mathrm{H}_{\text {artif-forêt }}\right)$ qui est maximum lorsqu'on a une répartition homogène entre les deux classes d'occupation du sol. Parmi les autres classes d'indices (diversité, hétérogénéité, connectivité), les indices interclasses forêt/urbain (moyenne sectionnale) et forêt/agricole (écart-type sectionnal) sont significatifs, ainsi que l'écart-type de l'indice de diversité de Shannon. L'écart-type de l'indice de diversité de Shannon est faible dans les sections cadastrales où la diversité varie peu entre les différentes 
fenêtres de $300 \times 300$ à l'intérieur de la section cadastrale. Les résultats présentent une appréciation positive de la stabilité dans la diversité des couverts d'une zone, qui indique qu'un paysage sous forme de mosaïque de taille moyenne monospécifique peut ne pas être apprécié. Cela invite à travailler plus avant sur les conséquences des échelles utilisées sur les prix implicites associés à la moyenne et à l'écart-type de l'indice de Shannon. On notera l'absence ${ }^{11}$ d'effet au sein de (M1) de l'indice d'agrégation (GAI) de l'indice de juxtaposition et d'interspection (GIJI) qui pâtissent de notre point de vue de la faible résolution des images LANDSAT. En revanche, ces indices deviennent significatifs, voire très significatif pour ce qui concerne l'indice de juxtaposition et d'interspection (GIJI), dès lors que l'on considère pas les autres variables renseignant le contexte socio-économique des communes et les variables d'accessibilité comme l'attestent les résultats du modèle (M2) présentés cidessous.

\section{[TABLEAU 4]}

\section{Conclusion}

Les résultats proposés ici permettent de valider une voie très fructueuse dans la prise en compte de l'hétérogénéité de l'espace dans les modèles hédoniques. Au sein des modèles standards centre/périphérie, la distance est supposée rendre compte de la distribution des autres éléments d'environnement sur le territoire. Nous observons ici qu'une meilleure prise en compte des formes fonctionnelles des caractéristiques continues des maisons et des éléments caractérisant l'environnement physique des biens conduit à des estimations beaucoup plus faibles des effets spécifiques de la distance au centre urbain dans une zone multipolaire comme celle du département des Bouches-du-Rhône. Si l'effet de localisation par rapport à Marseille joue indéniablement, les variables d'occupation du sol sont très significatives et permettent un meilleur ajustement du modèle dans les zones périurbaines et rurales. Ce type de données d'occupation du sol et de couvert est désormais disponible à grande échelle à une résolution tout à fait adaptée pour rendre compte d'un certain nombre d'hétérogénéités spatiales que les modèles monocentriques standards peinent encore à intégrer (WU et PLATINGA 2003).

Les résultats mitigés des grandes catégories d'indices (diversité, hétérogénéité, connectivité) dans leur capacité à rendre compte des variations de prix des maisons individuelles ne sont naturellement valables que pour les catégorisations de couverts et les données satellitales que nous avons utilisées. L'utilisation d'images de meilleure résolution, passant par l'intégration - actuellement en cours - dans nos modèles de données QUIKBIRD, permet d'envisager une catégorisation plus fine des couverts et une variabilité de ces indices plus en adéquation avec les observations de terrain. Notamment, l'indice de juxtaposition et d'interspection, lorsqu'il est exprimé à partir de statistiques renseignant sa distribution pour des fenêtres de $300 \times 300$ à l'intérieur d'une section cadastrale donnent des résultats très prometteurs et devraient nous permettre à terme de valider empiriquement les propositions de GEOGHEGAN et al. 1997.

Au-delà de la qualité des données utilisées, quelques difficultés méthodologiques demandent un travail spécifique. En premier lieu, certaines variables paysagères construites ne relèvent pas exclusivement de phénomènes reliés à la représentation des paysages, mais renvoie à des phénomènes sociaux plus complexes. Par exemple, la dominance (vue dominante, section cadastrale plus élevée que le noyau villageois) peut être interprétée comme un phénomène de distanciation sociale et largement structuré par un rattachement à des groupes sociaux particuliers. II serait donc intéressant d'estimer les modèles proposés en segmentant la population ou en instrumentalisant certaines variables socio-économiques.

Les problèmes d'endogénéité introduits par l'utilisation de variables paysagers doivent également être testés: le phénomène de mitage par exemple que l'on peut lire dans l'indice «tissu urbain discontinu » explique un part importante du prix des maisons dans certaines zones mais peut être surdéterminé par des variables utilisées comme explicatives des prix (structure du POS, distance au centre, composition sociale de la commune, ...). Se prémunir contre ce type d'endogénéité demanderait une réelle prise en compte des dynamiques de construction des paysages ; là encore, l'utilisation de variables instrumentales pourrait permettre de contourner ce problème.

\footnotetext{
${ }^{11}$ L'indice de diversité RPR étant très corrélé avec l'indice de diversité de Shannon, son absence ne peut pas permettre de statuer sur l'absence d'effet de la diversité des couverts.
} 


\section{Bibliographie :}

ANSELIN L., 1988, Spatial Econometrics : Methods and Models, Kluwer Academic Publishers, Dordrecht

ANSELIN L., BERA A. K., FLORAX R. et YOON M. J., 1996, "Simple diagnostic test for spatial dépendance", Regional Science and Urban Economics, vol. 26, pp. 77-104

BAUDRY J. et BUREL F., 2000, Ecologie du paysage : outils méthodes et concepts, Lavoisier, Paris

BELL K. et BOCKSTAEL N. E., 1997, An exemple of spatial economic modeling : land use conversion in Howard county, Maryland

BOLITZER B. et NETUSIL N. R., 2000, "The impact of open spaces on property values in Portland, Oregon", Journal of environmental and economics management, vol. 59, pp. 185-193

CAN A., 1990, "The measurement of neighbourhood dynamics in urban house prices", Economic Geography, vol. 66, pp. 254-272

CHOPPING M. et HAINES R. H., 1997, "Quantifying landscape structure: a review of landscape indices and their application to forested landscapes", Progress in Physical Geography, vol. 20, pp. 418-445

DAVIDSON R. et MACKINNON J. G., 2004, Econometric Theory and Methods, Oxford University Press, New York

DE COULON, 1988, Pourquoi un beau paysage est-il beau ? Essai sur l'esthétique du paysage, Office fédéral des Forêts et de la Protection du paysage, Berne

DUBIN R. A., 1998, "Spatial autocorrelation: a primer", Journal of Housing Economics, vol. 7, pp. 304327

FABUREL G., LAMBERT S. et SINDZINGRE A., 2001, Monétarisation des nuisances sonores routiuères et ferroviaires en milieu urbain, Rapport final pour la commission Monétarisation du PREDIT ( ADEEM, DRAST, Mission Bruit-Mate, RATP)

FABUREL G. et MALEYRE I., 2002, "Méthodes d'évaluation des impacts immobiliers", Etudes foncières, vol. 99, pp. 22-28

FISCHESSER B. et DUPUIS-TATE M. F., 2001, Politiques publiques et paysages. Du marché foncier à la lecture paysagère, Mimeo Cemagref, Grenoble

GARROD G. et WILLIS K., 1992, "The Environmental Economic Impact of Woodland: A Two-Stage Hedonic Price Model of the Amenity Value of Forestry in Britain", Applied-Economics, vol. 24, pp. 715728

GENIAUX G., NAPOLÉONE C., BOUILLON C., MORGE D., RAYMOND V., FISCHESSER B. et DUPUIS-TATE M. F., 2003, Du marché foncier à la lecture paysagère: Contribution à une méthodologie d'évaluation des valeurs paysagères en région PACA, Rapport pour le Ministère de l'aménagent du territoire et de l'environnement, CEMAGREF/INRA/IDEP

GEOGHEGAN J., WAINGER L. et BOCKSTAEL N., 1997, "Spatial land-scape indices in a hedonic framework: an ecological eco-nomics analysis using GIS", Ecological Economics, vol. 23, pp. 251-264

GÉRARD-VARET L. A., 2000, Les grands métropoles et la croissance urbaine : un examen de quelques enjeux de l'action publique, Note de recherche de l'IDEP NR-02-00, Marseille

IRWIN E. et BELL K., 2001, "Understanding and predicting patterns of urban growth at the rural-urban fringe using a spatially explicit model of residential land use change", en cours de publication, vol. pp.

KIEMSTEDT H., 1967, "Möglichkeiten zur Bestimmung der Erholungseignung in unterschiedlichen Landschaftsräumen", Natur und Landschaf, vol. 42, n¹1, pp. 243-248

LE CORBUSIER, 1971, La charte d'Athènes - 1933-1942, Seuil, Paris

LESVEQUES T. J., 1994, "Modelling the effects of airport noise on residential housing markets: A case study of Winnipeg International Airport", Journal of Transport Economics and Policy, vol. 28, $n^{\circ} 2$ pp. $199-210$ 
LUTTIK J., 2000, "The value of trees, water and open space as reflected by house prices in the Netherlands", Landscape and Urban Planning, vol. 48, n³-4, pp. 161-167

MORANCHO A. B., 2003, "A hedonic valuation of urban green areas", Landscape and Urban Planning, vol. 66, n`1, pp. 35-41

RONCAYOLO M., 1999, La ville et ses territoires, Folio, Paris

SMITH V. K., POULOS C. et KIM H., 2002, "Treating open space as an urban amenity", Ressource and Energy Economics, vol. 24, pp. 107-129

TYRVAINEN L., 1997, "The amenity value of the urban forest: an application of the hedonic pricing method", Landscape and Urban Planning, vol. 37, n³-4, pp. 211-222

WICKHAM J. D., O'NEILL R. V. et JONES K. B., 2000, "Forest fragmentation as an economic indicator", Landscape Ecology, vol. 15, pp. 171-179

WU J. et PLATINGA A. J., 2003, "The influence of public open space on urban spatial structure", Journal of Environmental Economics and Management, vol. 46, pp. 288-309 
Figure 1 : Exemple de croisement entre l'occupation du sol obtenue par photo-interprétation, les sections cadastrales et un quadrillage de $300 \times 300$ mètres (la photo de la commune est mise en référence pour une meilleure lecture).

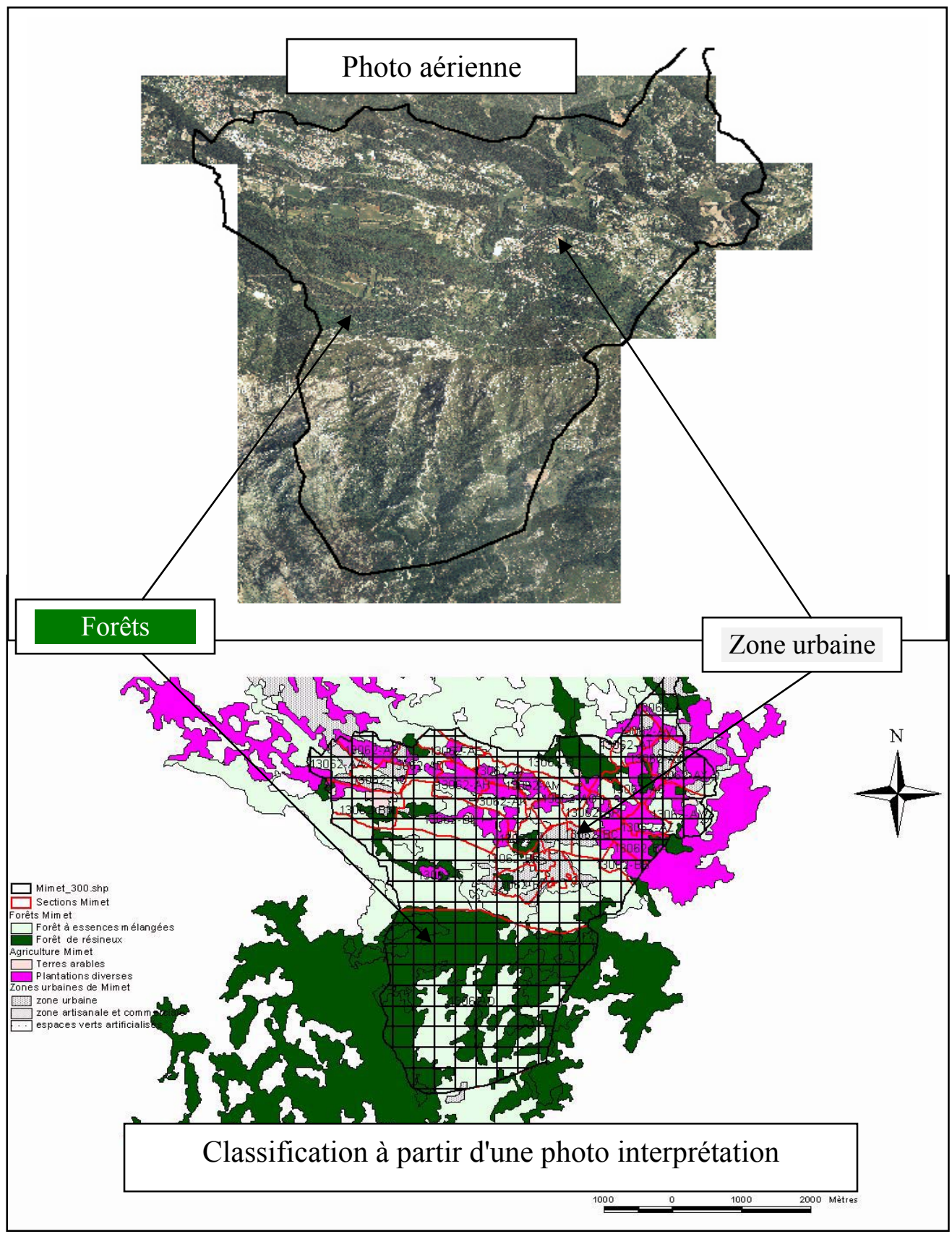


Tableau 1 : Données collectées et intégrées dans un Système d'Information Géographique

\begin{tabular}{|c|c|c|c|}
\hline $\begin{array}{c}\text { Nature des } \\
\text { Informations }\end{array}$ & Information apportée & Période ou zone & Sources \\
\hline Quantifiées & $\begin{array}{l}\text { Ventes de terres agricoles } \\
\text { Ventes de terres constructibles et } \\
\text { maisons individuelles } \\
\text { Recensement population } \\
\text { Fiscalité foncière }\end{array}$ & $\begin{array}{c}89 / 99 \\
91 / 2001 \\
99 \\
90 / 2000\end{array}$ & $\begin{array}{l}\text { SAFER } \\
\text { Chambre de notaires BDR } \\
\text { INSEE RGP } 99 \\
\text { DGI }\end{array}$ \\
\hline Numérisées & Sections cadastrales & BDR & Cemagref \\
\hline Spatiales & $\begin{array}{l}\text { Géographie physique } \\
\text { Voies de communication } \\
\text { Zonages Paysagers } \\
\text { POS } \\
\text { Indices écologie du paysage }\end{array}$ & $\begin{array}{l}\text { PACA } \\
\text { PACA } \\
\text { PACA } \\
\text { PACA } \\
\text { BDR } \\
\end{array}$ & $\begin{array}{l}\text { BD Carto GN } \\
\text { DRE } \\
\text { DIREN } \\
\text { CETE } \\
\text { Cemagref-IMEP }\end{array}$ \\
\hline
\end{tabular}


Tableau 2 : Estimation OLS (robuste à l'hétéroscédasticité) du modèle final sur l'ensemble des variables

\begin{tabular}{|l|c|c|c|c|}
\hline Source & DF & $\begin{array}{c}\text { Sum of } \\
\text { Squares }\end{array}$ & $\begin{array}{c}\text { Mean } \\
\text { Square }\end{array}$ & F \\
\hline Model & 75 & $1,253895 \mathrm{E} 15$ & $1,67186 \mathrm{E} 13$ & 477,41 \\
\hline Error & 13468 & $4,716366 \mathrm{E} 14$ & $3,5019 \mathrm{E}+10$ & $\mathrm{Pr}>\mathbf{F}$ \\
\hline $\begin{array}{l}\text { Corrected } \\
\text { Total }\end{array}$ & 13543 & $1,725532 \mathrm{E} 15$ & & $<.0001$ \\
\hline
\end{tabular}

\begin{tabular}{|l|c|l|c|}
\hline Racine MSE & 187134 & R2 & 0,7267 \\
\hline $\begin{array}{l}\text { Moyenne } \\
\text { dépendante }\end{array}$ & 2100772 & R2c & 0,7251 \\
\hline Coef Var & 8,90786 & Lambda & 0,31 \\
\hline
\end{tabular}

\begin{tabular}{|c|c|c|c|c|c|}
\hline VARIABLES & Paramètres & Ecart type & Chi2 & Pr > Chi2 & VIF \\
\hline Constante & -640206 & 27141 & 359,02 & $<.0001$ & 0 \\
\hline trend-annuel & 29676 & 643,12 & 1908,68 & $<.0001$ & 1,1805 \\
\hline Pas de terrain & 526355 & 18189 & 482,03 & $<.0001$ & 1,71478 \\
\hline Log(Surface du terrain) & 87378 & 1877,94 & 1471,82 & $<.0001$ & 2,29434 \\
\hline Log(Surface habitable) & 400547 & 5197,64 & 3332,49 & $<.0001$ & 1,52468 \\
\hline Type Maison Manquant & -42462 & 4325,14 & 76,11 & $<.0001$ & 1,40817 \\
\hline Type Maison Villa & 91593 & 5452,03 & 263,22 & $<.0001$ & 1,32045 \\
\hline Type Maison Divers & -247211 & 22544 & 61,44 & $<.0001$ & 1,05371 \\
\hline Epoque de construction Manquant & -47248 & 5261,75 & 70,11 & $<.0001$ & 1,17769 \\
\hline Epoque de construction 4 & -69575 & 5086,54 & 160,62 & $<.0001$ & 1,14608 \\
\hline Epoque de construction 3 & -118687 & 7713,61 & 165,89 & $<.0001$ & 1,19013 \\
\hline Epoque de construction 2 & -165228 & 8761,52 & 217,38 & $<.0001$ & 1,20292 \\
\hline Epoque de construction 1 & -142146 & 12228 & 61,78 & $<.0001$ & 1,15038 \\
\hline Plus d'une salle de bain & 44192 & 7798,15 & 24,90 & $<.0001$ & 1,05256 \\
\hline Place de parking & 63588 & 4868,53 & 150,61 & $<.0001$ & 1,28388 \\
\hline Piscine & 114691 & 10208 & 143,49 & $<.0001$ & 1,12435 \\
\hline Distance-Temps à Marseille & $-18,88984$ & 2,18 & 70,19 & $<.0001$ & 2,5446 \\
\hline Distance à l'autoroute le plus proche & 9681,33624 & 1734,79 & 29,43 & $<.0001$ & 1,46883 \\
\hline Imposition moyenne communal & 16,38456 & 0,53 & 684,14 & $<.0001$ & 2,58839 \\
\hline$\%$ de zone ND de la section & -28608 & 6179,63 & 18,17 & $<.0001$ & 1,18797 \\
\hline Orientation de la section & $-291,47227$ & 55,93 & 27,45 & $<.0001$ & 1,44549 \\
\hline La visibilité au-delà d'un rayon de $5 \mathrm{~km}$ (PVisi5000sup) & $-12,09638$ & 6,18 & 4,59 & 0,0322 & 1,13613 \\
\hline Ecart type sectionnal de l'indice de diversité Shannon ( $m S H D I)$ & 48475 & 21022 & 5,29 & 0,0215 & 1,39516 \\
\hline $\begin{array}{l}\text { Moyenne sectionnale de l'indice d'hétérogénéité interclasses } \\
\text { entre zones Artificialisées et Forêt ( } m H 13)\end{array}$ & 45677 & 18923 & 5,74 & 0,0166 & 1,46976 \\
\hline $\begin{array}{l}\text { Ecart type sectionnal de l'indice d'hétérogénéité interclasses } \\
\text { entre Territoire Agricole et Forêt }(s H 13)\end{array}$ & -70355 & 17619 & 15,59 & $<.0001$ & 1,7655 \\
\hline GPLAND : Tissu urbain continu & 2786,84408 & 867,29 & 11,73 & $<.0001$ & 1,48154 \\
\hline GPLAND : Bâti isolé & 604,1302 & 70,74 & 69,59 & $<.0001$ & 1,57766 \\
\hline $\begin{array}{l}\text { GPLAND : Zones industrielles ou commerciales et réseaux de } \\
\text { communication }\end{array}$ & $-361,47255$ & 132,76 & 7,11 & 0,0077 & 1,52956 \\
\hline GPLAND : Mines décharges et chantiers & $-909,33226$ & 284,97 & 12,28 & 0,0005 & 1,26846 \\
\hline $\begin{array}{l}\text { GPLAND : Territoires à dominante agricole avec présence } \\
\text { d'habitat résidentiel }\end{array}$ & 202,52931 & 71,53 & 6,77 & 0,0093 & 2,23137 \\
\hline GPLAND : Forêts & $-146,34847$ & 22,08 & 37,17 & $<.0001$ & 1,38513 \\
\hline commune $=13003$ & -135394 & 20413 & 39,21 & $<.0001$ & 1,08723 \\
\hline commune $=13005$ & 72678 & 10957 & 33,71 & $<.0001$ & 1,11358 \\
\hline commune $=13006$ & -79232 & 33235 & 3,88 & 0,0489 & 1,03836 \\
\hline commune $=13009$ & -148382 & 52182 & 25,36 & $<.0001$ & 1,00988 \\
\hline commune $=13013$ & -143767 & 33465 & 21,97 & $<.0001$ & 1,02095 \\
\hline commune $=13014$ & -201905 & 17779 & 107,68 & $<.0001$ & 1,04692 \\
\hline commune $=13015$ & -43110 & 12360 & 13,98 & 0,0002 & 1,19199 \\
\hline commune $=13018$ & -76782 & 31520 & 7,42 & 0,0065 & 1,04684 \\
\hline commune $=13019$ & -134122 & 15500 & 71,82 & $<.0001$ & 1,34511 \\
\hline commune $=13024$ & -89686 & 26099 & 9,15 & 0,0025 & 1,10405 \\
\hline commune $=13025$ & -91120 & 32856 & 6,98 & 0,0082 & 1,07611 \\
\hline commune $=13026$ & -41880 & 16091 & 8,34 & 0,0039 & 1,08957 \\
\hline commune $=13029$ & -154801 & 37331 & 10,28 & 0,0013 & 1,0327 \\
\hline commune $=13032$ & -54435 & 17826 & 6,85 & 0,0088 & 1,14166 \\
\hline commune $=13033$ & 43710 & 16660 & 5,52 & 0,0188 & 1,2531 \\
\hline commune $=13035$ & -31725 & 10178 & 9,94 & 0,0016 & 1,42176 \\
\hline commune $=13040$ & -98293 & 20239 & 26,27 & $<.0001$ & 1,03421 \\
\hline commune $=13043$ & -62797 & 14843 & 28,23 & $<.0001$ & 1,10506 \\
\hline commune $=13044$ & -128996 & 20203 & 35,33 & $<.0001$ & 1,06497 \\
\hline commune $=13046$ & 60313 & 23155 & 11,75 & 0,0006 & 1,03589 \\
\hline commune $=13047$ & -25573 & 9850 & 8,04 & 0,0046 & 1,25956 \\
\hline commune $=13050$ & -45440 & 18259 & 5,70 & 0,0169 & 1,06681 \\
\hline commune $=13054$ & -29169 & 8745,28 & 13,32 & 0,0003 & 1,22841 \\
\hline commune $=13060$ & -77560 & 26136 & 4,28 & 0,0386 & 1,02981 \\
\hline commune $=13064$ & -176389 & 37408 & 16,88 & $<.0001$ & 1,03698 \\
\hline commune $=13067$ & -169267 & 39533 & 8,04 & 0,0046 & 1,02472 \\
\hline commune=13069 & -60746 & 12350 & 22,68 & $<.0001$ & 1,08555 \\
\hline commune $=13072$ & -91226 & 25747 & 14,81 & $<.0001$ & 1,03688 \\
\hline commune $=13075$ & 82666 & 22190 & 19,52 & $<.0001$ & 1,04877 \\
\hline commune $=13076$ & -113576 & 42286 & 14,29 & 0,0002 & 1,0197 \\
\hline commune $=13077$ & -81647 & 17783 & 24,50 & $<.0001$ & 1,29539 \\
\hline commune $=13081$ & -61728 & 12021 & 45,12 & $<.0001$ & 1,07203 \\
\hline commune $=13082$ & -155546 & 23596 & 27,30 & $<.0001$ & 1,09145 \\
\hline commune $=13085$ & 57849 & 20809 & 8,16 & 0,0043 & 1,04441 \\
\hline commune $=13089$ & -150124 & 56841 & 7,26 & 0,0070 & 1,01405 \\
\hline commune $=13092$ & -93103 & 22990 & 15,03 & 0,0001 & 1,2749 \\
\hline commune $=13097$ & -56337 & 11874 & 27,75 & $<.0001$ & 1,42991 \\
\hline commune $=13098$ & -164769 & 20770 & 61,92 & $<.0001$ & 1,441 \\
\hline commune $=13101$ & 49016 & 23843 & 7,70 & 0,0055 & 1,03408 \\
\hline commune $=13105$ & -138184 & 17604 & 80,20 & $<.0001$ & 1,06993 \\
\hline commune $=13109$ & -158184 & 35781 & 12,46 & 0,0004 & 1,16712 \\
\hline commune $=13112$ & -36287 & 16599 & 5,00 & 0,0253 & 1,14402 \\
\hline commune $=13114$ & -103989 & 19459 & 18,57 & $<.0001$ & 1,16912 \\
\hline commune $=13115$ & -145061 & 34148 & 12,06 & 0,0005 & 1,02992 \\
\hline Regroupement de communes $\mathrm{n}^{\circ} 4$ (zone notariale) & 162524 & 8213,11 & 263,26 & $<.0001$ & 1,15184 \\
\hline
\end{tabular}


Tableau 3 : Tests de significativité par groupe de variables sur M1

\begin{tabular}{|l|c|c|c|c|}
\hline \multicolumn{1}{|c|}{ Groupe de variable } & $\begin{array}{c}\text { Type de } \\
\text { Variables }\end{array}$ & $\begin{array}{c}\text { Nombre de } \\
\text { variables }\end{array}$ & Chi2 & Pr $>$ ChiSq \\
\hline Année de vente & continue & 1 & 1908,68 & $<.0001$ \\
\hline Surface habitable & continue & 1 & 3332,49 & $<.0001$ \\
\hline Surface du terrain & continue & 2 & 1472,53 & $<.0001$ \\
\hline Type de Maison & muette & 4 & 457,22 & $<.0001$ \\
\hline Epoque de construction & muette & 5 & 478,27 & $<.0001$ \\
\hline Autres caractéristiques de la maison & muette & 3 & 343,98 & $<.0001$ \\
\hline Zone de marché & muette & 1 & 263,26 & $<.0001$ \\
\hline Commune & muette & 44 & 719,49 & $<.0001$ \\
\hline POS & continue & 1 & 18,17 & $<.0001$ \\
\hline Richesse de la commune & continue & 1 & 684,14 & $<.0001$ \\
\hline Variables de distance & continue & 2 & 93,63 & $<.0001$ \\
\hline Variables paysagères à dire d'expert & continue & 2 & 30,20 & $<.0001$ \\
\hline Variables d'occupation du sol & continue & 6 & 145,02 & $<.0001$ \\
\hline Indices écologie du paysage & continue & 3 & 21,07 & $<.0001$ \\
\hline Toutes variables paysagères & continue & 11 & 226,40 & $<.0001$ \\
\hline
\end{tabular}

Tableau 4: Estimation OLS (robuste à l'hétéroscédasticité) du modèle (M1) avec comme variables d'environnement uniquement les variables paysagères.

\begin{tabular}{|c|c|c|c|c|c|}
\hline VARIABLES & Paramètres & Ecart type & Chi2 & $\mathrm{Pr}>\mathrm{Chi2}$ & VIF \\
\hline Constante & 256369 & 203505 & 1,21 & 0,2713 & 0 \\
\hline trend-annuel & 24946 & 718,09 & 1106,32 & $<.0001$ & 1,12018 \\
\hline Pas de terrain & 542959 & 20560 & 445,68 & $<.0001$ & 1,65848 \\
\hline Surface du terrain & 87488 & 2072,88 & 1286,86 & $<.0001$ & 2,12852 \\
\hline Surface habitable & 418724 & 5682,99 & 3398,20 & $<.0001$ & 1,40991 \\
\hline Type Maison Manquant & -37934 & 4962,24 & 47,86 & $<.0001$ & 1,41102 \\
\hline Type Maison Villa & 130724 & 6114,11 & 424,80 & $<.0001$ & 1,28808 \\
\hline Type Maison Divers & -214804 & 25510 & 32,24 & $<.0001$ & 1,05035 \\
\hline Epoque de construction Manquant & -34652 & 6062,20 & 28,34 & $<.0001$ & 1,14063 \\
\hline Epoque de construction 4 & -52582 & 5761,41 & 68,59 & $<.0001$ & 1,11317 \\
\hline Epoque de construction 3 & -97653 & 8758,50 & 89,10 & $<.0001$ & 1,16531 \\
\hline Epoque de construction 2 & -164194 & 10048 & 177,99 & $<.0001$ & 1,21928 \\
\hline Epoque de construction 1 & -156320 & 13959 & 63,08 & $<.0001$ & 1,15180 \\
\hline Plus d'une salle de bain & 33861 & 8850,28 & 11,85 & $<.0001$ & 1,04359 \\
\hline Place de parking & 76863 & 5513,83 & 175,11 & $<.0001$ & 1,26907 \\
\hline Piscine & 114761 & 1152 & 109,68 & $<, 0001$ & 1,11414 \\
\hline Visibilité au-delà d'un rayon de 5 km (PVisi5000sup) & 137,63225 & 51,36 & 6,76 & 0,0093 & 1,17877 \\
\hline GAl indice d'agrégation & 2109,13618 & 2028,27 & 4,28 & 0,0386 & 2,13876 \\
\hline GIJI Indice d'interspection et de juxtaposition & $-4762,28152$ & 217,12 & 99,83 & $<.0001$ & 1,55841 \\
\hline Moyenne sectionnal de SHDI & -185090 & 19151 & 83,02 & $<.0001$ & 2,76546 \\
\hline $\begin{array}{l}\text { Moyenne sectionnale de l'indice d'hétérogénéité interclasses entre } \\
\text { zones Artificialisées et Forêt }(m H 13)\end{array}$ & 83334 & 18518 & 19,08 & $<.0001$ & 1,31263 \\
\hline $\begin{array}{l}\text { Ecart type sectionnal de l'indice d'hétérogénéité interclasses entre } \\
\text { Territoire Agricole et Forêt ( }(\mathrm{H} 13)\end{array}$ & 108338 & 21655 & 25,56 & $<.0001$ & 1,46261 \\
\hline PLAND : Tissu urbain continu & $-5585,01011$ & 1087,62 & 26,83 & $<.0001$ & 1,82931 \\
\hline PLAND : Tissu urbain discontinu & $-480,38109$ & 93,40 & 23,59 & $<.0001$ & 2,44084 \\
\hline \multirow{3}{*}{$\begin{array}{l}\text { PLAND : Bâti isolé } \\
\text { PLAND : Zones industrielles ou commerciales et réseaux de } \\
\text { communication } \\
\text { PLAND : Mines décharges et chantiers }\end{array}$} & 1752,29229 & 80,17 & 504,59 & $<.0001$ & 1,61096 \\
\hline & $-684,82182$ & 153,19 & 12,91 & 0,0003 & 1,61957 \\
\hline & $-1692,45393$ & 303,49 & 36,03 & $<.0001$ & 1,15016 \\
\hline PLAND : Territoires agricoles & $-101,15467$ & 20,38 & 12,53 & 0,0004 & 1,18847 \\
\hline PLAND : Forêts & $-274,89874$ & 22,90 & 132,19 & $<.0001$ & 1,18793 \\
\hline PLAND : Espaces ouverts sans ou avec peu de végétation & $-133,54794$ & 41,06 & 13,59 & 0,0002 & 1,34755 \\
\hline
\end{tabular}


Tableau 5: Tests spatiaux sur M1

\begin{tabular}{|c|c|c|c|c|c|c|c|c|}
\hline \multicolumn{2}{|c|}{ Matrice de poids } & I & ZI & Pratio & LMerr & LMerr* & LMlag & LMlag* \\
\hline \multirow{3}{*}{$\begin{array}{l}\text { contiguité } \\
\text { communale }\end{array}$} & intra-communale & $-0,007047$ & $-0,720631$ & 0,2355682 & 0,8420773 & 0,907296 & 0,8230345 & 0,8882532 \\
\hline & intercommunale & $-0,004098$ & $-0,916581$ & 0,1796812 & 1,2123583 & 1,253063 & 0,5450937 & 0,5857984 \\
\hline & intercommunale ordre2 & $-0,00435$ & $-1,783318$ & 0,0372673 & 3,3639437 & 3,4551192 & 1,2822227 & 1,3733982 \\
\hline \multirow{3}{*}{$\begin{array}{l}\text { inverse de la } \\
\text { distance }\end{array}$} & sans seuil & $-0,001052$ & 0,0654362 & 0,4739133 & 0,2203143 & 0,2836146 & 3,6888763 & 3,7521765 \\
\hline & avec seuil $20 \mathrm{~km}$ & $-0,001626$ & $-0,07247$ & 0,4711139 & 0,2480029 & 0,306091 & 3,211865 & 3,2699531 \\
\hline & avec seuil $10 \mathrm{~km}$ & $-0,000964$ & 0,1352256 & 0,4462168 & 0,0429834 & 0,0750424 & 3,5249343 & 3,5569932 \\
\hline \multirow{3}{*}{ Voisinage } & à moins de $5 \mathrm{~km}$ & $-0,012738$ & $-2,018894$ & 0,0217491 & 4,6472155 & 4,785927 & 0,8593096 & 0,998021 \\
\hline & à moins de $10 \mathrm{~km}$ & & & & & & & \\
\hline & à moins de $20 \mathrm{~km}$ & $-0,00261$ & $-0,990122$ & 0,1610573 & 1,6704989 & 1,7130688 & 0,7826489 & 0,8252188 \\
\hline
\end{tabular}

Tableau 6: Tests spatiaux sur un modèle sans variable d'environnement $\mathrm{M} 0: P(\lambda)=\alpha+\beta X_{C}+\varepsilon$

\begin{tabular}{|c|c|c|c|c|c|c|c|c|}
\hline \multicolumn{2}{|c|}{ Matrice de poids } & I & ZI & Pratio & LMerr & LMerrstar & LMlag & LMlagstar \\
\hline \multirow{3}{*}{$\begin{array}{l}\text { contiguité } \\
\text { communale }\end{array}$} & intra-communale & 0,1742202 & 23,785695 & 0 & 514,66001 & 511,80079 & 4,7193158 & 1,8600877 \\
\hline & intercommunale & 0,0906532 & 26,980762 & 0 & 593,38901 & 595,91161 & 3,7918295 & 6,3144317 \\
\hline & ordre2 & 0,0394601 & 20,461712 & 0 & 276,80991 & 278,05657 & 2,7585874 & 4,0052446 \\
\hline \multirow{3}{*}{$\begin{array}{l}\text { inverse de la } \\
\text { distance }\end{array}$} & sans seuil & 0,0373427 & 21,192576 & 0 & 277,85865 & 278,61709 & 0,267038 & 1,0254754 \\
\hline & avec seuil 20 km & 0,0587305 & 21,094595 & 0 & 323,6826 & 324,88162 & 0,8931251 & 2,0921459 \\
\hline & avec seuil $10 \mathrm{~km}$ & 0,0919428 & 21,791121 & 0 & 391,34966 & 392,1639 & 0,1034051 & 0,9176378 \\
\hline \multirow{3}{*}{ Voisinage } & à moins de 5 km & 0,0875563 & 15,908037 & 0 & 219,54939 & 221,74862 & 4,9780456 & 7,1772736 \\
\hline & à moins de $10 \mathrm{~km}$ & & & & & & & \\
\hline & à moins de $20 \mathrm{~km}$ & 0,0083842 & 6,8505538 & $3,678 \mathrm{E}-12$ & 17,232348 & 17,658617 & 9,1502682 & 9,5765367 \\
\hline
\end{tabular}


Figure 2 : distribution spatiale des résidus, modèle final en bas (M1) et modèle avec uniquement les caractéristiques des maisons en haut (M0).

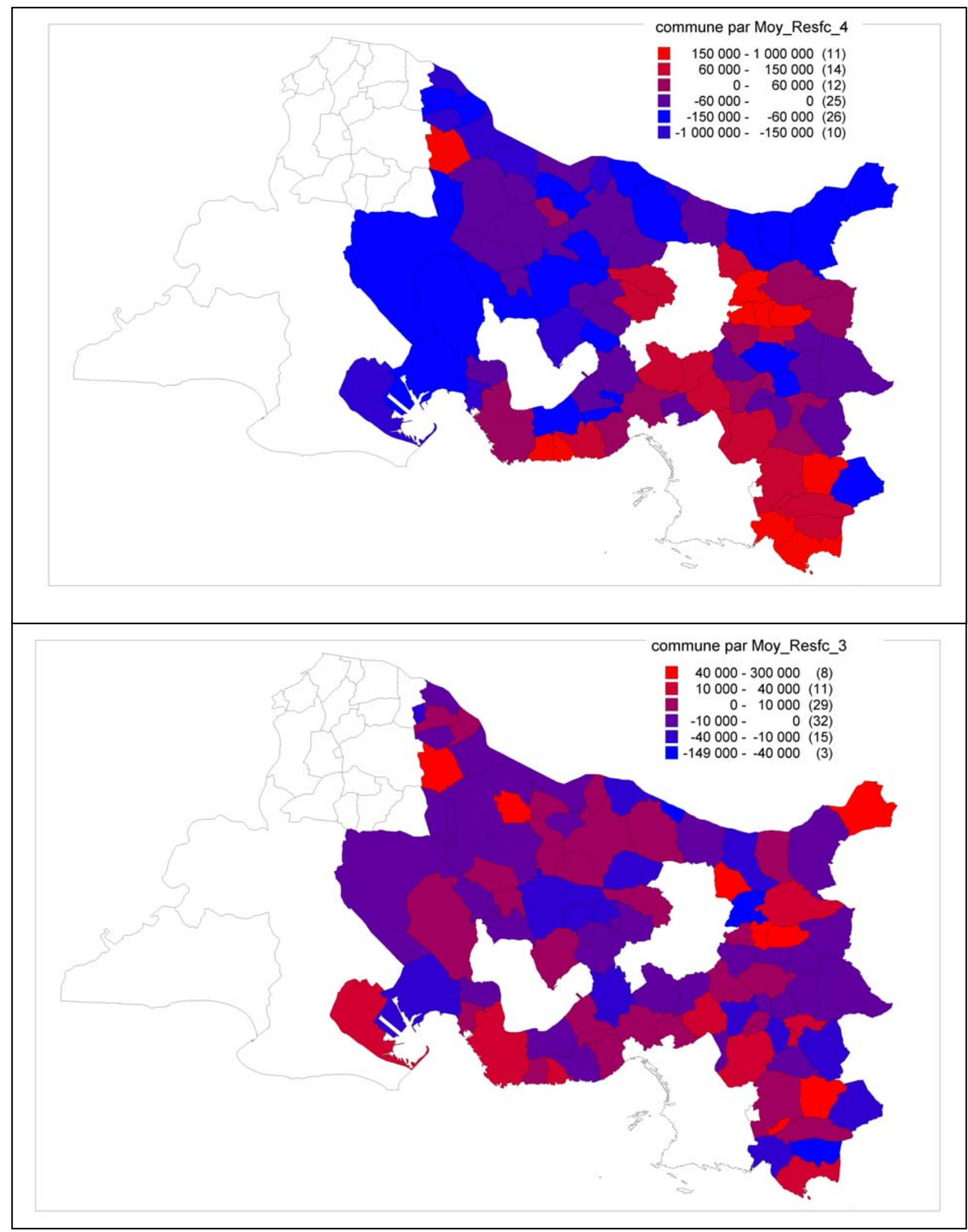

